\title{
La división de la filosofía práctica en Kant
}

Roberto Casales García Universidad Popular Autónoma del Estado de Puebla / Universidad Nacional Autónoma de México

Resumen

La filosofía práctica de Kant constituye un todo sistemático cuyas propuestas se organizan o estructuran en tres grandes disciplinas: en primer lugar, encontramos lo que bien puede denominarse como moral pura a priori, la cual contiene el fundamento último de la moralidad y, por ende, de la racionalidad práctica; en segundo lugar, está una metafísica de las costumbres, cuya misión es la de contener el sistema de las leyes de la razón práctica, y, finalmente, la antropología práctica, disciplina que nos da todos aquellos datos empíricos que favorecen u obstaculizan el desarrollo moral de los seres humanos.

Palabras clave: moral, ley moral, Immanuel Kant, metafísica de las costumbres, antropología.

\section{Abstract}

Kant's practical philosophy costitute a systematic whole whose proposals are organized or structured in three major disciplines: in first place, we find what may be denominated as pure ethics, which con- 
tains the ultimate ground of morality and, therefore, of the practical rationality; in second place, there is a metaphysics of morals, whose mission is to contain the system of laws of the practical reason, and, finally, practical anthropology, a discipline that give us every empirical knowledge that favor or hinder moral development of human beings.

Keywords: Moral, Moral law, Immanuel Kant, Metaphysics of morals, Anthropology.

\section{La estructura general de la filosofía práctica kantiana}

La filosofía práctica de Kant, como toda su filosofía, manifiesta peculiar. Desafortunadamente, la estructura de la filosofía práctica de Kant no ha sido ampliamente desarrollada. Esto representa un grave problema para el estudio general de la filosofía práctica de Kant. Ya que al omitir su estructura, se corre el riesgo de hacer una interpretación parcial de la filosofía práctica de nuestro autor $\mathrm{y}$, en consecuencia, caer en una visión formalista o rigorista de la misma. En efecto, interpretaciones como la formalista o la rigorista -según las cuales la filosofía práctica de Kant se reduce a una deontología meramente formal sin considerar ningún aspecto empírico, o bien, a una filosofía práctica cuya realización de la moral, siendo esta imperativa, no contempla las emociones, inclinaciones, etc., sino sólo el deber por sí mismo (aus Pflicht)- tienen en común que parten sólo de uno de los elementos que conforman la filosofía práctica kantiana.

Teniendo esto en mente, es importante hacer notar que Kant mismo no explicitó de forma plena la estructura de la filosofía práctica, como sí hizo con los saberes de la razón en su uso especulativo al dilucidar la arquitectónica de la razón pura (cf. $K r V$, A 832 / B 860-A 852 / B 880) y el esquema general de las ciencias 
de la naturaleza (cf. $M A, 477-479$ ). Sin embargo, contamos con algunos pasajes de sus obras en los que fundamenta esta estructura, los cuales nos servirán de guía.

El primer indicio de esta división o estructura de la filosofía práctica lo encontramos en la Fundamentación..., donde Kant nos dice: "La filosofía griega se dividía en tres ciencias: la física, la ética y la lógica. Esta división es perfectamente adecuada a la naturaleza de la cuestión y no hay en ella nada que mejorar, a no ser, acaso, solamente ańadir el principio de la misma, en parte para asegurarse de esta manera de que es completa y en parte para poder determinar correctamente las subdivisiones" (GMS, 387 1-8). La filosofía en general, entendida como sistema, se divide primero en su parte formal y en su parte material, "de las cuales la primera (la lógica) comprende meramente la forma de pensar, en un sistema de reglas, y la segunda (la parte real) somete sistemáticamente a consideración los objetos sobre los cuales se piensa, en la medida en que sea posible un conocimiento racional de los mismos a partir de conceptos" $(E E, 1)$.

La filosofía meramente formal es la lógica, puesto que versa sobre las leyes universales y necesarias del pensar, mientras que la parte material de la filosofía se constituye por la física y la ética, ya que los objetos sobre los que versa (la parte material de la filosofía) siguen dos tipos de leyes (Gesetze), a saber: leyes de la naturaleza (Gesetze der Natur) o leyes de la libertad (Gesetze der Freiheit) (cf. $G M S, 387,11-15) 1$. Las leyes de la primera pertenecen a la física, mientras que las leyes de la segunda pertenecen a la moral,

${ }^{1}$ Sobre esta división entre leyes de la naturaleza y leyes de la libertad, es impor-
tante recordar lo siguiente: "Las leyes de la naturaleza constituyen los objetos
naturales o teóricos, mientras que las leyes morales constituyen los objetos de la
libertad o prácticos. Puesto que se trata de legislaciones independientes, se habla
de dos objetos distintos, pero no debe olvidarse que, para Kant, objeto no es
lo mismo que realidad. En el caso de la acción humana esto es particularmente
claro, pues una misma acción se puede (y se debe) considerar de acuerdo con las 
una como doctrina de la naturaleza y la otra como doctrina de las costumbres (cf. GMS, 387, 15-17). De esta primera división de la filosofía en general, vemos dos cosas: en primer lugar, que la filosofía entendida como sistema se divide en filosofía teórica y en filosofía práctica, tal y como sostendrá posteriormente en la Primera introducción a la Critica de la facultad de juzgar (cf. EE, 1; Moralphilosophie Collins 27:243), y, en segundo lugar, que la estructura de la doctrina de la naturaleza y la ética es semejante. Una primera similitud entre ellas radica en que ambas, a diferencia de la lógica2, tienen una parte empírica. Tanto la filosofía de la naturaleza como la filosofía moral necesitan de ésta, "porque aquella tiene que determinar sus leyes para la naturaleza como un objeto de la experiencia, y ésta para la voluntad del hombre, en tanto que es afectada por la naturaleza” (GMS, 387, 22-27).

De la misma forma, ambas cuentan con una parte racional, "la que presenta sus doctrinas exclusivamente a partir de principios a priori” (GMS, 388, 5-6). Esta última si es meramente formal se llama lógica, mientras que si considera algunos objetos y está determinada por ellos, se llama metafísica (cf. GMS, 388, 7-10). "La metafísica se divide en la [metafísica] de uso especulativo de la razón pura, y en la de uso práctico de ella, y es, por tanto, o bien metafísica de la naturaleza (Metaphysik der Natur), o bien metafísica de las costumbres (Metaphysik der Sitten)" (KrV, A 841 / B 869). Tanto la filosofía de la naturaleza como la ética, así, tienen una parte racional y una empírica.

Dentro de esta moral racional cuyos fundamentos son a priori, cabe una división más, la cual parte de la distinción que se estable-

leyes naturales, en cuanto fenómenos, así como efecto de la libertad, según la ley moral" (Torralba, 2011: 22).

${ }^{2}$ Según Kant, la lógica no puede tener una parte empírica, ya que de tenerla no podría ser "un canon para el entendimiento o la razón que vale en todo pensar y tiene que ser demostrado" (GMS, 387, 17-21). 
ce entre el saber puro a priori y el saber impuro a priori. Normalmente se suele confundir lo puro y lo a priori, debido a dos cosas: en primer lugar, a que Kant a veces usa de forma ambigua ambas expresiones $y$, en segundo lugar, a que todo aquel conocimiento que es puro es, al mismo tiempo, a priori. No obstante, lo a priori y lo puro son dos cosas distintas.3 "Entre los conocimientos a priori llámense puros aquellos en los que no está mezclado nada empírico. Así, por ejemplo: la proposición toda mudanza tiene su causa, es una proposición a priori, pero no pura, porque mudanza es un concepto que sólo puede ser extraído de la experiencia” ( $\mathrm{Kr} V$, B 3).4 En el caso de la filosofía práctica, podríamos decir que, por ejemplo, la primera formulación del Imperativo Categórico es un principio puro a priori, mientras que los preceptos de la antroponomía son principios que siendo a priori, necesitan de un dato empírico. "[L]a filosofía de la razón pura es, o bien propedéutica (ejercicio preliminar), que investiga la facultad de la razón con respecto a todos los conocimientos puros a priori, y se llama crítica, o bien, en segundo término, [es] el sistema de la razón pura (ciencia), el completo conocimiento filosófico (tanto verdadero como

${ }^{3}$ Una de las interpretaciones de la filosofía práctica de Kant que manifiesta claramente esta distinción en el terreno de lo práctico es la de David Ross, a pesar de ser una interpretación polémica y reducir la moral kantiana a la Fundamentación... y a la Crítica de la razón práctica -esto es, a la moral pura-. Dicha interpretación nos dice, por ejemplo, que el principio "no debes mentir" es un principio a priori, más no puro a priori, ya que éste "contiene un elemento obtenido de la experiencia" (Cf. Ross, 1965: 2).

${ }^{4}$ Según Torralba: "Tanto en la teoría del conocimiento como en la teoría de la acción, Kant reconoce que hay una "doble raíz": intelectual y sensible, pura y empírica. Esta doble raíz da lugar a una distinción entre la facultad y su objeto, o entre la voluntad y sus fines" (Torralba, 2011: 22). En este sentido, la teoría kantiana de la acción tendrá dos niveles distintos: primero, una teoría de la acción general, donde no se distingue entre el imperativo categórico y el hipotético; segundo, la moral propiamente dicha, donde lo que rige es la ley moral. 
aparente) por razón pura, en interconexión sistemática, y se llama metafisica" (KrV, A 841 / B 869).

Aquella parte de la filosofía práctica que se caracteriza por fundarse en principios a priori tiene dos elementos: el primero consolida el sistema de la crítica de la razón práctica, concebida como una propedéutica la metafísica de lo práctico que se caracteriza por investigar "la capacidad de la razón respecto de los juicios puros a priori que versan sobre lo que debe ocurrir por libertad, o, con otras palabras," según nos dice Rovira, "como la indagación de las condiciones de posibilidad de los deberes morales" (Rovira, 1992: 80-81); el segundo es "el sistema de la ciencia práctica -o metafísica de lo práctico- [que] puede describirse como el conjunto de los juicios que tratan de lo que debe ocurrir por libertad, o sea, como el elenco ordenado de los deberes morales" (Rovira, 1992: 81). De esta forma, la filosofía práctica de Kant consistirá en tres partes: en primera instancia, una propedéutica o sistema de la crítica de la razón práctica, en la cual podemos ubicar de forma perfecta tanto a la Critica de la razón práctica como a la Fundamentación ...; en segunda instancia, una metafísica de lo práctico o de las costumbres, donde ubicamos a la obra del mismo nombre; finalmente, la antropología práctica o antropología moral, en la que podemos ubicar, como trataré de demostrar, la Antropología en sentido pragmático.

\section{Moral pura a priori: el sistema crítico y su fundamentación}

La moral pura a priori o propedéutica de la metafísica de lo práctico es, quizás, una de las partes de la filosofía práctica de Kant más estudiadas desde su gestación. Desafortunadamente, como ya mencioné reiteradas veces, ésta es una de las partes de la moral kantiana que frecuentemente se ha malinterpretado -ya sea como 
formalista, rigorista, etc. - y a la que constantemente se reduce la filosofía práctica de nuestro autor. Frente a estas lecturas parciales de la filosofía práctica de Kant es importante tener claro cuáles son los objetivos y las especificidades de esta primera parte de la filosofía práctica de Kant. Para lo cual nos preguntamos "si no se cree que es de la más extrema necesidad elaborar de una vez una filosofía moral pura que estuviese completamente limpia de todo cuanto sea empírico y pertenece a la antropología” (GMS, 389, 6-9).

El filósofo de Königsberg se inclinaría a pensar que la idea de deber no puede extraerse ni de una generalización de las experiencias sensibles ni del deseo de placer, sino a priori de conceptos de la razón pura (cf. Green, 1992: 176). Un principio que se apoya en fundamentos empíricos a lo más que podría aspirar es a ser una regla práctica, más nunca una ley moral. Sin una moral pura, los principios prácticos sólo lograrían tener una base o fundamento contingente y la ley moral no podría justificar de manera correcta qué máximas 5 pueden considerarse moralmente buenas, o permisibles, y cuáles malas, o prohibidas.

${ }^{5}$ Es relevante recordar que una ley práctica (praktische Gesetz) y una máxima (Maxime) son dos cosas distintas, ya que una ley práctica es un principio objetivo del querer y, por tanto, es válido para todo ser racional, mientras que una máxima es un principio meramente subjetivo. En este sentido debe entenderse lo que Kant nos dice en la Crítica de la razón práctica: "Principios fundamentales prácticos son las proposiciones que contienen una determinación universal de la voluntad, a la cual se subordinan varias reglas prácticas. Son subjetivos o máximas si la condición es considerada por el sujeto como válida sólo para su voluntad; en cambio, son objetivos o leyes prácticas si la condición es reconocida como objetiva, es decir, válida para la voluntad de todo ser racional" ( $K p V$, 19). La ética kantiana, tal y como nos lo señala la Dra. Dulce María Granja, es una "ética de máximas" (Cf. Granja, 2010: 107-144). Una de las razones para afirmar esto la encontramos en un artículo de Gustavo Leyva, donde nos dice: "en la argumentación kantiana el paso constructivo hacia la formulación de leyes válidas de modo universal y necesario no puede ser realizado sin recurrir a las máximas como principios subjetivos de acción si es que esas leyes universales 
De esta forma, la moral pura a priori no toma nada de la experiencia: sus fundamentos descansan enteramente en ella. Esto no significa, sin embargo, que la filosofía práctica de Kant haga caso omiso de la experiencia y del conocimiento de la naturaleza del hombre, pues los principios morales "exigen además una capacidad de juzgar aguzada por la experiencia, en parte para distinguir en qué casos tienen su aplicación, y en parte para procurarles acceso en la voluntad del hombre y energía para la ejecución" (GMS, 389, 30-33)-; por el contrario, significa que la moral se funda en principios enteramente racionales. La ley moral en su pureza y autenticidad, lo cual es lo más importante de lo práctico a los ojos del filósofo de Königsberg, sólo se puede encontrar en la filosofía pura.

La moral pura sirve como preparación o propedéutica para la metafísica de las costumbres. Ésta, sin embargo, no debe ser confundida con una filosofía práctica universal (allgemeinen praktiktischen Weltweisheit) de Wolff, la cual "no sometió a consideración una voluntad de tipo especial, por ejemplo una voluntad que sea determinada sin ningún motivo empírico, completamente por principios a priori, y a la que se podría denominar pura, sino el querer en general con todas las acciones y condiciones que le convienen en ese significado universal" (GMS, 390, 24-29). Esto mismo refuerza la distinción que debe hacerse entre el sistema crítico de la razón pura práctica y la filosofía práctica en general, ya que si bien una contempla todas las partes relativas a la acción y, en ese sentido, es general, la moral pura investiga "la idea y los principios de una voluntad pura (reinen Willens) posible y no las acciones y condiciones del querer humano en general (die Handlungen und Bedingungen des menschlichen), las cuales en su mayor parte se extraen de la psicología (Psychologie)" (GMS, 390, 34-391, 1) .

y necesarias han de ejercer un influjo sobre la determinación de la voluntad del sujeto que actúa" (Leyva, 2008: 325). 
El sistema de la crítica de la razón pura estudia el fundamento último por el cual tanto una máxima como una acción pueden ser juzgadas según su valor moral. "Así, la Fundamentación... nos proporciona un análisis de la conciencia ordinaria que tiene como punto de partida los juicios morales comunes y el sentimiento de la obligatoriedad del deber y que busca poner de manifiesto los orígenes de tales juicios" (Granja, 1996: 138). ${ }^{6}$ Esta parte de la filosofía práctica de Kant pregunta por aquello que es incondicionalmente bueno, esto es, aquello que es bueno sin restricción, pues sólo en virtud de aquello que es incondicionalmente bueno, todo aquello que se dice bueno de forma restringida, puede ser bueno. En resumidas cuentas, la moral pura "no es nada más que la búsqueda y establecimiento del principio supremo de la moralidad (des obersten Prinzips der Moralität), lo cual constituye por sí solo un quehacer aislado, entero en su propósito, y que ha de ser separado de toda otra investigación moral" (GMS, 392, 3-7).

\section{Moral impura a priori: la metafísica de las costumbres}

De la misma forma en la que a la Crítica de la razón pura les sigue una Metafísica de la naturaleza, al sistema crítico de la razón práctica debe seguirle una metafísica de las costumbres (Methaphysik

${ }^{6} \mathrm{La}$ fundamentación parte del conocimiento ordinario de la moral al conocimiento filosófico: "en el primer apartado se parte del 'conocimiento moral común de la razón (gemeine sittliche Vernunfterkenntniss)', es decir, de la comprensión habitual, por así decirlo prefilosófica, de la moralidad para, sobre esta base, acceder 'analíticamente (analytisch)', en el segundo apartado, a la 'determinación del principio supremo (zur Bestimmung des obersten Princips)' de la moralidad, esto es, a la 'metafísica de las costumbres (Metaphysik der Sitten)'. Finalmente, en el tercer apartado, Kant intenta establecer un puente 'desde la metafísica de las costumbres hacia la razón práctica pura (von der Metaphysik der Sitten zur Kritik der reinen praktischen Vernunft)"' (Leyva, 2008: 327-328). 
der Sitten) (cf. $M d S, 205) .^{7}$ Si bien la primera parte de la filosofía práctica de Kant se estudia el principio supremo de la moralidad, la metafísica de las costumbres tendrá por objeto de estudio el sistema de las leyes de la razón pura práctica. "Estas leyes son, sin embargo, al decir de Kant, de dos tipos principales: o bien son tales que no sólo prescriben una acción como deber, sino que, a la vez,

${ }^{7}$ Sobre este respecto, Mary J. Gregor nos dice que la metafísica de las costumbres comprende a toda la moral racional, esto es, tanto a la propedéutica como a la metafísica de lo práctico (Cf. Gregor, 1960: 239-240). Esto, sin embargo, se debe a la ambigüedad que hay en el uso que Kant mismo hace del término "puro". "La dificultad se origina en la falla al observar, en la práctica, la distinción que él dibujó entre 'conocimiento puro' y 'conocimiento a priori'” (Cf. Gregor, 1960: 241). Por esta razón, Kant mismo usa de forma ambigua el término "metafísica", el cual, por una parte, hace alusión a todo aquel conocimiento racional, ya sea puro o meramente a priori, y, por otra, a aquel conocimiento que siendo a priori, contiene una parte mínima de experiencia.

Con la finalidad de salvar la ambigüedad y distinguir la parte pura de la parte impura, Mary J. Gregor rescata la distinción que Kant, refiriéndose al ámbito especulativo, hace entre "metafísica trascendental" y "metafísica de la naturaleza corpórea” (Cf. $M A, 470-472$ ). La primera, es decir, la parte trascendental, estudia los principios y conceptos a priori que hacen posible nuestra experiencia de los objetos en general y, según Gregor, está contenida en la primera Crítica (Cf. Gregor, 1960: 246). Por el contrario, la metafísica de la naturaleza de lo corpóreo contiene aquello que puede ser conocido a priori considerando un elemento empírico, a saber, la naturaleza corpórea o material de los objetos que estudia (Cf. $M A, 472$ ). Esta misma distinción aplicada a la moral nos daría dos partes de la metafísica de las costumbres: una parte trascendental, que versa sobre el principio supremo de la moralidad como ley para los seres racionales en general y una segunda parte que trata sobre cómo las leyes de la conducta humana pueden ser determinadas al aplicar el principio supremo de la moral a un limitado conocimiento empírico del hombre (Cf. Gregor, 1960: 248).

Por lo que este trabajo de investigación compete, reservaré el término "metafísica de las costumbre" única y exclusivamente para la segunda parte de la filosofía práctica, mientras que a la parte pura le llamaré según los diversos sinónimos con los que hemos caracterizado a ésta. Esto nos ayudará a tener mayor claridad expositiva y, al mismo tiempo, salvaguardar las ambigüedades en el uso de dichos términos. 
convierten también a este deber en motivo del obrar; o bien son tales que no incluyen al deber como motivo del obrar. Las primeras son leyes morales; las segundas, leyes jurídicas" (Rovira, 1992: 83). Estos principios de la acción, en cuanto tal, son principios que entran dentro de la categoría de conocimiento a priori, ya que "la conexión de sujeto y predicado es hecha por la razón independientemente de la experiencia” (Gregor, 1960: 241). Sin embargo, tal y como Mary J. Gregor señala, estos principios no son puros, pues parte de sus conceptos es dada por la experiencia (cf. Gregor, 1960: 241). "Estas leyes de la libertad (Freiheit), a diferencia de las leyes de la naturaleza, se llaman morales. Si afectan sólo a acciones meramente externas y a su conformidad con la ley, se llaman jurídicas; pero si exigen también que ellas mismas (las leyes) deban ser los fundamentos de determinación de las acciones, entonces son éticas" ( $M d S, 214)$.

Según Kant, la primera división de la moral como sistema de los deberes en general, esto es, de la metafísica de las costumbres, es:

División de la Moral como Sistema de los deberes en general

Doctrina elemental

Deberes jurídicos Deberes de virtud

Derecho Privado

Derecho público

Esquema 1. $(M d S, 242)^{8}$

${ }^{8}$ Esta es la primera división que Kant ofrece respecto a la estructura interna de la metafísica de las costumbres, no obstante, ésta sólo será un esquema general al cual hay que agregar las distinciones entre deberes que posteriormente va intro- 
Así, la metafísica de las costumbres, en cuanto que consolida la segunda parte de la filosofía práctica de Kant, se dividirá en dos secciones: una primera doctrina del derecho (Retchtslehre) y una doctrina de la virtud (Tugendlehre). "La libertad a la que se refieren las primeras leyes sólo puede ser la libertad en el ejercicio externo del arbitrio, pero aquella a la que se refieren las últimas puede ser la libertad tanto en el ejercicio externo como en el interno del arbitrio, en tanto que está determinado por leyes de la razón" (MdS, 214). ' Estas leyes, como mencionamos anteriormente, se fundan como a priori y necesarias. En este sentido, la metafísica de las costumbres no es una doctrina de la felicidad, pues en una doctrina de este tipo: "Todo aparente razonamiento a priori no es aquí, en el fondo, más que experiencia, elevada a generalidad por inducción; generalidad (secundum principia generalia, non universalia) que además es tan precaria que ha de permitirse a cada uno una infinidad de excepciones para adaptar la elección de un modo de vida a su peculiar inclinación y a su predisposición hacia el placer, y para escarmentar, por último, sólo en cabeza propia o en la ajena" ( $M d S, 215-216)$.

Por el contrario, los preceptos morales mandan a cada uno sin atender a sus inclinaciones, pues cada hombre es libre y está dotado de razón práctica. La enseñanza de las leyes de la razón práctica

duciendo. Por ahora no me detendré en esto, pues podría alejarnos de nuestro objetivo principal.

${ }^{9}$ Sobre este respecto, Kant agregará: "En la filosofía teórica se dice: en el espacio están sólo los objetos del sentido externo, pero en el tiempo están todos, tanto los objetos del sentido externo como los del interno: porque las representaciones de ambos son sin duda representaciones y, en esta medida, pertenecen en su totalidad al sentido interno. De igual modo, consideramos la libertad en el uso externo o interno del arbitrio, sus leyes, como leyes puras prácticas de la razón para el arbitrio libre, tienen que ser a la vez fundamentos internos de determinación del mismo, aunque no siempre sea posible considerarlas bajo este aspecto" (MdS, 214). 
no se toma de la observación de uno mismo ni de la propia animalidad. Tampoco se toma de la percepción del curso del mundo, esto es, de lo que sucede y cómo se obra, "sino que la razón manda cómo se debe obrar, aun cuando no se encontrara un ejemplo todavía de ello" (MdS, 216). La moral no descansa en ninguno de estos aspectos, sino que, a lo más, "se sirve de ellos (como consejos) sólo como un contrapeso frente a las seducciones contrarias, para compensar de antemano el vicio de una ponderación parcial en el juicio práctico y para asegurar a éste ante todo una ponderación acorde con el peso de los principios a priori de una razón pura práctica" (MdS, 216). A este sistema de conocimientos a priori se le llama, según Kant, metafísica de lo práctico. Esta, sin embargo, requiere un conocimiento empírico mínimo que, en palabra de Mary Gregor, es: "el hombre considerado simplemente como hombre" (Gregor, 1960: 248). La metafísica de las costumbres, como la segunda parte de la filosofía práctica de Kant, aun cuando tiene una parte empírica mínima, no puede admitir el conocimiento de las circunstancias contingentes ni las condiciones en las cuales el hombre puede encontrarse. "Ella determinará, por tanto, leyes morales válidas para todo hombre meramente en virtud de su naturaleza humana” (Gregor, 1960: 248).

Una moral impura a priori, tal y como la hemos caracterizado a lo largo de este apartado, contiene en sí todos aquellos principios para aplicar el principio supremo de la moral a la experiencia, para lo cual toma frecuentemente como objeto la naturaleza peculiar del hombre, la cual sólo es cognoscible por la experiencia. Sólo por medio de ambas podemos mostrar las consecuencias de los principios morales universales, "sin disminuir por ello, sin embargo, la pureza de los últimos, ni poner en duda su origen a priori” ( $M d S$, 217). Igualmente, esto significa que, si bien la metafísica de las costumbres no puede fundamentarse en la antropología, sí puede aplicarse a ella, tal y como señalaré a lo largo del próximo apartado. 


\section{Antropología y carácter moral}

A diferencia de una moral pura y de la metafísica de las costumbres, la antropología práctica o moral se caracteriza por ser un conocimiento empírico del hombre y su especificidad. Uno de los primeros problemas al que nos enfrentamos cuando hablamos de una antropología moral en la filosofía práctica de nuestro autor es, tal y como señala Claudia Schmidt (2005: 159-166), la diversidad de proyectos antropológicos relativos a su filosofía. Según Schmidt, podemos distinguir al menos cuatro proyectos antropológicos, a saber: la antropología trascendental, la empírica, la pragmática y la moral (Schmidt, 2005: pp. 157-159). La antropología trascendental debe considerarse aquella que engloba las grandes preguntas de la filosofía. En palabras de Kant:

El campo de la filosofía en esta significación cosmopolita se puede reducir a las siguientes preguntas:

1) ¿Qué puedo saber?

2) ¿Qué debo hacer?

3) ¿Qué tengo derecho a esperar?

4) ¿Qué es el hombre?

A la primera pregunta contesta la metafísica, a la segunda la moral, a la tercer la religión, y a la cuarta la antropología. Pero en el fondo se podría considerar todo esto en la antropología, pues las tres primeras preguntas se refieren a la última (Jäsche Logik, 25).

El problema que suscita el análisis de Schmidt está en que ella distingue la Antropología en sentido pragmático, como un mero estudio empírico del hombre, de la antropología práctica, a la que Kant refiere tanto en la Fundamentación... como en la Metafísica de las costumbres. Para ella, la Antropología en sentido pragmático contiene sólo una antropología empírica, entendiendo por ésta una suerte de psicología que, a pesar de estudiar las causas de las acciones humanas, es ajena a la moral. De la misma forma, ella lla- 
ma antropología pragmática a aquel estudio del hombre que, por una parte, investiga el desarrollo del individuo y, por otra, indica que el individuo humano tiene una predisposición pragmática a usar otras personas hábilmente para sus propósitos personales. Finalmente, Claudia Schmidt sostendrá que la antropología moral es única y exclusivamente la antroponomía, esto es, los principios para la aplicación del principio supremo de la moralidad al caso específico del hombre (cf. Schmidt, 2005: 176; Wilson, 1997: 88). Esta distinción, sin embargo, irá en contra de la descripción que Kant, en la Metafísica de las costumbres, hace de la antropología práctica o moral, la cual, en palabras de nuestro autor, estudia "las condiciones subjetivas, tanto obstaculizadoras como favorecedoras, de la realización de las leyes de la primera en la naturaleza humana, la creación, difusión y consolidación de los principios morales (en la educación y en la enseñanza escolar y popular) y de igual modo otras enseñanzas y prescripciones fundadas en la experiencia” ( $M d S, 217)$. Bajo esta descripción de la antropología, Kant nos señalará que la tercera parte que conforma su filosofía práctica es un complemento de las dos anteriores.

No me detendré en este debate, sin embargo es importante señalar dos argumentos por los cuales sostengo que la antropología moral está contenida en el último texto publicado por Kant. La primera razón nos remite a la historia del texto, la cual inicia en los cursos de Antropología práctica impartidos por el filósofo de Königsberg desde el semestre de invierno de 1772-1773 y concluidos con la publicación de la Antropología en sentido pragmático, obra que mantiene muchas semejanzas con los distintos manuscritos presentados por sus alumnos. Uno de los manuscritos que más llama nuestra atención es el que Mrongovius publicó en 1785, mismo año de publicación de la Fundamentación de la metafísica de las costumbres. Lo cual nos hace pensar que la ciencia antropológica en la Kant pensaba al momento de escribir esta última obra 
no era otra que la misma antropología que impartía en sus cursos (vid. Rodríguez, 2004: IX-XXI; Stark, 2003: 15-37). El segundo argumento lo expone Mary J. Gregor diciendo: "La antropología moral debe distinguirse de la parte de la ética que aplica la ley moral a ciertos tipos de situaciones y de tal modo determina los deberes del hombre en ciertas circunstancias. Sin importar qué tanto conocimiento empírico puedan contener estas reglas, ellas siguen siendo principios morales a priori. No obstante, los preceptos derivados de la antropología moral "se basan sobre la experiencia" (Gregor, 1960: 243). Así, la diferencia entre una antroponomía y la antropología práctica radica justo en que la primera es a priori, a pesar de que toma como objeto el conocimiento de la naturaleza del hombre (conocimiento que es meramente empírico), mientras que la segunda, siendo meramente empírica, sólo es susceptible de ser orientada por principios a priori.

Ahora bien, el conocimiento antropológico (Menschenkenntnis), según Kant, puede hacerse de dos formas: en sentido fisiológico o en un sentido pragmático (cf. $A p H, 119) .{ }^{10}$ Una antropología en sentido fisiológico estudia lo que la naturaleza hace del hombre, una antropología en sentido pragmático investiga "lo que él, como ser que obra libremente, hace, o puede y debe hacer, de sí mismo" (ApH, 119). El conocimiento pragmático de esta ciencia exige que éste sea un conocimiento del hombre como ciudadano del mundo (Welt). Esto mismo se puede ver con mayor claridad en las expresiones conocer el mundo y tener mundo, "pues el que conoce el mundo se limita a comprender el juego que ha presenciado, mientras que el que tiene mundo ha entrado en juego en él" $(A p H, 120)$. Esta tercera parte de la filosofía práctica de Kant, así, no se conforma con ser un mero conocimiento del mundo, sino

${ }^{10}$ Dada la discusión anterior, utilizaré el término pragmático para designar la antropología moral, aunque teniendo en cuenta que no todo el contenido de la Antropología en sentido pragmático es relevante a la moral de forma directa. 
que es, en cierto sentido, una philosophia moralis applicata. La cual, a pesar de sus dificultades propias, tiene la ventaja de ser una ciencia que fomenta y acelera su crecimiento con la ayuda de todos aquellos amantes de esta ciencia. En palabras de Kant:

Una antropología sistemáticamente concebida y, sin embargo, popularmente (haciendo referencia a ejemplos que cualquier lector pueda encontrar al respecto) desarrollada en sentido pragmático, lleva consigo la ventaja para el público lector de que gracias a la completitud de los títulos bajo los cuales puede colocarse esta o aquella cualidad humana observada y concerniente a la práctica, se le proporciona a este público el mismo número de ocasiones e incitaciones para hacer de cada cualidad en particular un tema propio, para colocarla en el departamento que le corresponda; con lo cual los trabajos en esta antropología se repartirán por sí mismas entre los amantes de su estudio y se reunirán poco a poco en un todo, por obra de la unidad del plan: con lo cual, a su vez, se fomentará y acelerará el crecimiento de esta ciencia de utilidad común (ApH, 121-122).

Una antropología pragmática, al mismo tiempo que contiene una cierta doctrina de la prudencia, alude a un conocimiento del hombre que es provechoso al desarrollo moral del individuo. "Nosotros requerimos, en breve, una antropología práctica o moral, aquella parte de la ética que contiene las doctrinas y prescripciones basada en la experiencia (1) como las condiciones subjetivas que obstaculizan o promueven la ejecución de la ley (de la moral racional) en la naturaleza humana, y (2) la generación, propagación y fortalecimiento de los principios morales" (Munzel, 1999: 61). Parte fundamental que sirve a estos fines se encuentra en la segunda parte de la Antropología en sentido pragmático, la cual se titula Característica Antropológica.

En esta sección, el filósofo de Königsberg nos habla del carácter en dos significaciones: la primera alude al carácter físico, la segun- 
da al carácter moral. "El primero es el signo distintivo del hombre como ser sensible o natural; el segundo lo distingue como un ser racional, dotado de libertad. El varón de principios, de quien se sabe seguro lo que se ha de esperar, no por parte de su instinto, sino de su voluntad, tiene carácter" $(A p H, 285) .{ }^{11}$ Con esto en mente, Kant divide la característica del hombre en tres: a) lo natural o predisposiciones naturales, b) el temperamento y c) el carácter. De las cuales, las dos primeras indican lo que el hombre puede hacerse y sólo la última, que es propiamente moral, indica "lo que él está pronto a hacer de sí mismo" $(A p H, 285)$. La primera parte nos habla de todo aquello que conviene al hombre para un fin determinado, lo cual "Supone la capacidad de un hombre para aprender y la vocación de la naturaleza para el uso de los talentos" (AP Mrongovius, 99). Por su parte, el temperamento puede ser considerado desde dos perspectivas: una fisiológica y otra psicológica. Desde la primera perspectiva, el temperamento alude a la constitución y complexión corporales, ambos aspectos relativos a los fluidos y la fuerza vital en el hombre. En cambio, la perspectiva psicológica, que examina a éste como temperamento del alma, representa "aquellas expresiones tomadas a la cualidad de la sangre

${ }^{11}$ Sobre este último punto, deben tener en cuenta lo que Kant nos dice en la primera sección de la Fundamentación..., a saber: "En ningún lugar del mundo, pero tampoco siquiera fuera del mismo, es posible pensar nada que pudiese ser tenido por bueno sin restricción (Einschränkung), a no ser únicamente una buena voluntad (ein guter Wille). El entendimiento, el ingenio, la capacidad de juzgar, y como quiera que se llamen los demás talentos (Talente) del espíritu, o el buen ánimo, la decisión, la perseverancia en las intenciones, como propiedades del temperamento (Temperament), son, sin duda, en diversos respectos, buenos y deseables, pero también pueden llegar a ser en extremo malos y nocivos si la voluntad que ha de hacer uso de estos dones naturales, y cuya peculiar constitución se llama por eso carácter (Charakter), no es buena" (GMS, 393, 5-14). En este pasaje es claro que la concepción de la voluntad (Wille) y el carácter (Charakter) se relacionan íntimamente, pues no podría haber una voluntad buena si uno antes no se ha forjado en sí mismo un buen carácter. 
simplemente como inspiradas en la analogía del juego de los sentimientos y apetitos con causas motrices corporales (entre las cuales es la sangre la más destacada)" $(A p H, 286)$. De la que obtenemos cuatro tipos de temperamentos: el sanguíneo, el melancólico, el colérico y el flemático.

Si bien tanto el temperamento como lo natural son indispensables a la moral, en cuanto que el hombre está influido por ellos en su acción, todos ellos necesitan interactuar con el carácter en su índole moral para poder ser considerados buenos. "Poder decir de un hombre simplemente: "tiene un carácter», significa, no sólo decir mucho de él, sino también alabarle mucho; pues se trata de algo raro que suscita el respeto y la admiración" ( $A p H, 291-292)$. Tener un carácter, ${ }^{12}$ sea este bueno o malo, es tener aquella propiedad de la voluntad en virtud de la cual "el sujeto se vincula a sí mismo a determinados principios prácticos que se ha prescrito irrevocablemente por medio de su propia razón" $(A p H, 292)$. De esta forma, el carácter es un elemento esencial de la moral que está por encima de todo lo que la naturaleza le otorga al hombre. A diferencia del temperamento y lo natural que son dados por naturaleza, el carácter se forja por la voluntad conforme a principios, haciendo del hombre con carácter alguien que suscita respeto y admiración. "No se trata aquí de lo que la naturaleza hace del hombre, sino de lo que éste hace de sí mismo; pues lo primero es cosa del tempera-

${ }^{12}$ Kant nos había hablado de un "carácter trascendental" desde la Crítica de la razón pura (Cf. KrV, A 539/ B567), el cual alude a un aspecto del "Yo trascendental" en tanto a lo práctico. No obstante, dejaré éste de lado para referirme al carácter exclusivamente en un sentido práctico, el cual debe ser forjado con el tiempo, con las decisiones concretas siempre contextualizadas, en la confrontación con las inclinaciones sensibles, etc. Aunque posteriormente mencionaré algunas características del carácter trascendental para aclarar las nociones de Denkungsart y Gesinnung. Sobre el tema del carácter trascendental, confrontar Allison (1999: 29-53). 
mento (en que el sujeto es en gran parte pasivo), y únicamente lo último permite ver que tiene carácter" $(A p H, 292)$.

Con estos conceptos en mente, es claro que la Antropología en sentido pragmático, al menos en parte significativa, contiene en sí la antropología práctica de la que Kant habla en la Fundamentación... y en la Metafísica de las costumbres. Especialmente el tema del carácter práctico, propio de la antropología, adquiere una vital importancia para la filosofía práctica de nuestro autor. La cual sólo puede ser entendida si se observa la estructura de la filosofía práctica kantiana y, de la misma manera, se comprenden los puentes que unen a cada uno de sus elementos con los otros dos.

\section{De la moral pura a la antropología y a la inversa}

Una vez que conocemos los elementos que conforman la estructura de la filosofía práctica de Kant, es de vital importancia hablar acerca del tipo de relación que mantienen entre sí. Esto equivale a preguntarnos: ¿qué tipo de relación existe entre la moral pura, la metafísica de las costumbres y la antropología práctica? Una primera respuesta sería la que radicaliza la distinción a tal grado que hace de cada elemento de la filosofía práctica de nuestro autor una ciencia inconexa con las otras, de modo que no existe relación alguna. La segunda respuesta mantendrá una relación unidireccional, donde la relación va de la moral pura a la antropología, pues es claro, según lo visto, que la filosofía práctica de Kant sólo puede fundarse desde una filosofía pura. Finalmente, la tercera respuesta, hacia la cual me inclino, apuntará a una relación de bidireccionalidad, es decir, que no sólo va de la moral pura a la antropología en su fundamentación, sino también a la inversa. La primera respuesta puede respaldarse con lo que Kant mismo menciona en la Fundamentación... respecto a la división entre estas disciplinas: 
Si bien sería por sí mismo un objeto no indigno de consideración preguntar si la filosofía pura no reclama en todas sus partes un especialista, y si no sería mejor para el conjunto del oficio erudito si se advirtiese a quienes están acostumbrados, en conformidad con el gusto del público, a vender lo empírico mezclado con lo racional según todo tipo de proposiciones desconocidas para ellos mismos, a quienes se denominan a sí mismos pensadores independientes, y elucubradores a otros que preparan la parte meramente racional, que no cultiven a la vez dos quehaceres que son absolutamente distintos en la manera de tratarlos, para cada uno de los cuales se exige quizá un talento especial y cuya unión en una persona produce sólo chapuceros, con todo esto, aquí pregunto sin embargo sólo si la naturaleza de la ciencia no exige separar siempre cuidadosamente la parte empírica de la racional y hacer preceder a la física propiamente dicha (empírica) una metafísica de la naturaleza, y a la antropología práctica una metafísica de las costumbres, que tendrían que estar cuidadosamente limpias de todo lo empírico, para saber cuánto puede rendir la razón pura en ambos casos y de qué fuentes extrae ella su enseñanza a priori (GMS, 388, 22-399, 2).

Dentro este pasaje es clara la necesidad de saber distinguir las formas de tratar cada una de estas disciplinas, pues sus principios son distintos. No obstante, esto no significa que no exista un vínculo entre ellas, sino que es necesario saber diferenciar sus métodos y contenidos para poder estudiar cada una. La filosofía, según Kant, se distingue del conocimiento racional "en que presenta en ciencia separada lo que la segunda sólo concibe como mezclado” (GMS, 390, 15-16). Quien mezcla los principios de una moral pura, por ejemplo, con su parte empírica rompe la pureza misma de las costumbres y va en contra de su propio fin. A pesar de que es necesario hacer un estudio independiente de cada una de las partes de la filosofía práctica, para hacer filosofía es indispensable establecer "una conexión sistemática de esos conocimientos, o enlace de los 
mismos en la idea de un todo" (Jäsche Logik, 24). De este modo, aun cuando la filosofía práctica de nuestro autor exige que cada ciencia sea estudiada por separado, sin confundir ni mezclar los conocimientos de una con los de otra, ella exige que sus saberes contengan una unidad sistemática. Así, la primera respuesta es inviable e iría en contra del afán sistemático y arquitectónico de Kant.

La filosofía práctica del filósofo de Königsberg exige un hilo conductor (Leitfaden) en virtud del cual sus distintas partes adquieren sentido. ${ }^{13}$ El hilo conductor de la filosofía práctica de nuestro autor no puede ser otro sino una facultad de juzgar y une el saber

${ }^{13}$ En la Crítica de la razón pura, Kant usa esta expresión para hablar sobre el hilo conductor (Leitfaden) para descubrir todos los conceptos puros del entendimiento (Cf. $K r V$, A 66 / B 91-A 84 / B 116). Este término, al mismo tiempo que señala la necesidad de una clara distinción de los conceptos puros del entendimiento, reclama una cierta unidad sistemática. Esta unidad sistemática de los conceptos puros del entendimiento es dada por la facultad de juzgar, ya que "En cada juicio hay un concepto que vale por muchos, y bajo este muchos comprende también una representación dada, la cual, última, es referida entonces inmediatamente al objeto... Todos los juicios son, según esto, funciones de la unidad entre nuestras representaciones, pues en ellos, el conocimiento del objeto, se emplea, en lugar de una representación inmediata, una más elevada que comprende bajo sí a aquella y otras muchas; y en virtud de eso, muchos conocimientos posibles son recogidos en uno" ( $K r V$, A 68 / B 93-A 69 / B 94). No me detendré a ahondar más en esta temática, sino que buscaré una aplicación de este término a la filosofía práctica.

En la aplicación del término "Leitfaden" a la filosofía práctica de Kant, vemos que es útil a la temática que estamos tratando, ya que sirve para establecer la unidad sistemática entre sus tres partes. Al igual que en la filosofía teórica, el hilo conductor de la filosofía práctica sería la facultad de juzgar, bajo el matiz de que ésta es una facultad de juzgar libre. Este Leiffaden brinda una unidad sistemática a nociones como las de "buena voluntad", "bueno irrestricto", "ley moral", "deber", "Imperativo Categórico", "Virtud", "deber", "antroponomía”, "carácter", por mencionar algunos de los conceptos clave de la filosofía práctica de nuestro autor. 
práctico, entendiendo por práctico tanto aquello que inmediatamente determina a la voluntad como todo aquello que es posible mediante la libertad (Torralba, 2009: 34-40). De esta manera, las distintas partes que conforman la filosofía práctica de Kant no sólo mantienen una relación, sino que ésta debe tomar forma como una unidad sistemática, la cual no puede ser unidireccional, ya que ello implicaría una reducción al formalismo con el que frecuentemente se critica a la filosofía práctica de Kant. Un formalismo de este tipo, al mismo tiempo que eliminaría toda posible aplicación de la moral kantiana a la especificidad del hombre, haría imposible el concepto mismo de una metafísica de las costumbres. En efecto, si el tipo de relación que mantienen éstas es unidireccional, yendo sólo de la moral pura a la antropología, entonces los imperativos propios de la moral (aquellos que imperan de forma categórica) no podrían tener principios subordinados para su aplicación, ya que éstos toman "frecuentemente como objeto la naturaleza peculiar del hombre, cognoscible sólo por la experiencia" ( $M d S$, 217).

Una relación unidireccional, del mismo modo, hace estériles todas aquellas observaciones de la antropología práctica y, en consecuencia, no sería posible deducir de ellas ninguna primicia para la aplicación del principio supremo de la moral. Asimismo, no se podría determinar qué deberes nos corresponden en cada caso en concreto, ya que "Existen reglas respecto de las cuales, de hecho, hay que hacer excepciones para que en determinados casos alcancen su finalidad" (Reflexionen, 7214), las cuales sólo pueden ser dadas a partir de la experiencia. La parte racional de la filosofía práctica -esto es, la moral pura y la metafísica de las costumbres-y su parte empírica "están cercanamente relacionadas, y la moral no puede existir sin la antropología, pues uno primero debe conocer del agente si está en una posición para alcanzar lo que es requerido que deba hacer" (Moralphilosophie Collins, 27: 244). Si se sostiene una relación de tipo unidireccional, la filosofía práctica de Kant no 
tendría un lugar para la creatividad moral, así como tampoco para la formación de nuevos conceptos morales. Esto implicaría dos cosas: en primer lugar, que la función del Imperativo Categórico sólo es derivativa y determinante, y, en segundo, que la moral kantiana sería utópica e irrealizable por completo.

La relación entre las distintas partes de la filosofía práctica de Kant, por tanto, debe ser entendida bidireccionalmente. De este modo, no sólo se va de la moral pura a la antropología, sino también de la antropología a la moral pura. Es decir, no sólo se va de la pureza de la ley a lo empírico en su fundamentación, sino que también va de lo particular dado en una experiencia a lo universal en su aplicación.

\section{Bibliografía}

Tabla de abreviaturas de obras citadas de Immanuel Kant

$A p H$

Kant, Immanuel, 2014, Antropología en sentido pragmático, Dulce María Granja Castro y Gustavo Leyva (trads.), México, FCE/UAM/ UNAM.

$A P$

,2004, Antropología práctica. (Según el ma-

Mrongovius nuscrito inédito de C.C. Mrongovius, fechado en 1785), Roberto Rodríguez Aramayo (trad.), Madrid, Tecnos.

$K p V$ , 2005, Crítica de la razón práctica, Dulce María Granja Castro (trad.), México, FCE/ UAM/UNAM.

$\mathrm{KrV} \longrightarrow$, 2009, Crítica de la razón pura, Mario Caimi (trad.), México, FCE/UAM/UNAM. 
GMS

1999, Fundamentación de la metafísica de las costumbres, José Mardomingo (trad.), Barcelona, Ariel.

Jäsche Logik 2010, Lógica (Immanuel Kants Logik. Ein Handbuch zu Vorlesungen), Carlos Correa (trad.), Buenos Aires, Corregidor.

$M d S$ , 2005, Metafísica de las costumbres, Adela Cortina Orts y Jesús Collin Sancho (trads.), Madrid, Tecnos.

Moralphilosophie , 2001, "Moral Philosophy Collins", en LecCollins

$E E$ tures on ethics, Peter Heath y J. B. Schneewind (eds.), Peter Heath (trad.), Cambridge, Cambridge University Press.

, 1991, "Primera versión de la introducción a la Crítica de la facultad de juzgar", en Crítica de la facultad de juzgar, Pablo Oyarzún (trad.), Caracas, Monte Ávila.

$M A$ 1993, Primeros principios metafísicos de la ciencia de la naturaleza, Samuel Nemirovsky, México, UNAM.

Reflexionen , 2004, Reflexiones sobre filosofía moral, José G. Santos Herceg (trad.), Salamanca, Sígueme.

Fuentes secundarias

Allison, Henry, 1999, Kant's Theory of Freedom, Cambridge, Cambridge University Press.

Frierson, Patrick R., 2003, Freedom and Anthropology in Kant's Moral Philosophy, Cambridge, Cambridge University Press.

Granja, Dulce María, 1996, "El juicio reflexivo en la ética kantiana”, Diánoia. Anuario de Filosofía, vol. XLII, núm. 42, pp. 125-144. 
UAM.

2010, Lecciones de Kant para hoy, México, Antrophos I

Gregor, Mary J., 1960, “Kant's Conception of a `Metaphysic of Morals'", The Philosophical Quarterly, vol. 10, núm. 40, pp. 238-251.

Green, T. H., 1992, "The Good Will”, en Immanuel Kant. Critical Assessments, vol. I, , London/Nueva York, Routledge, pp. 162169.

Leyva, Gustavo, 2008, “Immanuel Kant: la razón de la acción”, en Filosofia de la acción. Un análisis histórico-sistemático de la acción y la racionalidad práctica en los clásicos de la filosofía, Madrid, Síntesis, pp. 315-363.

Louden, Robert, 2002, Impure Ethics, New York, Oxford University Press.

Mautner, Thomas, 1972, "Kant's Grundlegung", The Philosophical Quarterly, vol. 22, núm. 88, pp. 255-257.

Munzel, Felicitas, 1999, Kant's Conception of Moral Character: The "Critical" Link of Morality, Anthropologie, and Reflective Judgment, Chicago, The University of Chicago Press, pp. 377.

Rodríguez Aramayo, Roberto, 2004, "Kant ante la razón pragmática. (Una excursión por los bajos del deber ser)", en Antropología práctica. (Según el manuscrito inédito de C.C. Mrongovius, fechado en 1785), Madrid, Tecnos, pp. IX-XXVI.

Ross, David, 1965, Kant's Ethical Theory. A Commentary on the Grundlegung zur Metaphysik der Sitten, Oxford, Oxford University Press.

Rovira, Rogelio, 1992, "Las divisiones de la filosofía práctica de Kant", Anales del seminario de Historia de la Filosofía, núm. 9, pp. 79-87. 
Schmidt, Claudia M., 2005, "Kant's Trascendental, Empirical, Pragmatic, and Moral Anthropology", en Kant-Studien 96, Walter de Gruyter, Jahrg, pp. 156-182.

Stark, Werner, 2003, "Historical Notes and Interpretative Questions about Kant's Lectures on Anthropology", en Essays on Kant's Anthropology, Cambridge, Cambridge University Press, pp. 15-37.

Torralba, José María, 2011, "La teoría kantiana de la acción. De la noción de máxima como regla autoimpuesta a la descripción de la acción", Tópicos, núm. 41, pp. 17-61.

, 2009, Libertad, objeto práctico y acción. La facultad del juicio en la filosofia moral de Kant, Hildesheim, Georg Olms Verlag.

Recibido: 11 de agosto de 2014 Aceptado: 11 de noviembre de 2014 